\title{
OPTIMIZING BUDGET ALLOCATION AMONG PROJECT ACTIVITIES
}

\author{
Dimitri Golenko-Ginzburg', Zohar Laslo², Avner Ben-Yair² and Anna Baron ${ }^{3}$ \\ ${ }^{1}$ Department of Industrial Engineering and Management \\ Academic College of Judea and Samaria, Ariel, 44837,E-mail: dimitri@bgumail.bgu.ac.il \\ ${ }^{2}$ Department of Industrial Engineering and Management \\ Sami Shamoon College of Engineering, Beer-Sheva, 84100,Israel,Email: zohar@sce.ac.il / avnerb@sce.ac.il \\ ${ }^{3}$ Department of Industrial Engineering and Management, \\ Ben-Gurion University of the Negev, Beer-Sheva, 84105, Israel, E-mail: malishev@bgumail.bgu.ac.il
}

Received 3 October 2005; accepted 7 December 2006

\begin{abstract}
The problems of optimal budget allocation among project's activities are considered. The formulated optimization models and their solution can be used in planning, as well as in control and monitoring of individual projects in modern design offices.

Models of budget allocation for PERT-COST type networks, are considered as well.

The problem is to schedule a realization of all project's activities in order to accomplish the project on time, i.e., not later than the given due date, with minimal expenses, taking into account limitations on dynamics of resources consumption during the scheduled period. The solution is based on decomposition principle, i.e., decomposing a network project into network fragments, replacing them by a small number of aggregated activities, solving the problem for aggregated network project, and disaggregating the network diagram in order to schedule each project's activity.
\end{abstract}

Keywords: budget, network project, individual projects.

\section{Introduction}

The problems of optimal budget allocation among project's activities are considered. The formulated optimization models and their solution can be used in planning, as well as in control and monitoring of individual projects in modern design offices.

The problem is to schedule a realization of all project's activities in order to accomplish the project on time, i.e., not later than the given due date, with minimal expenses, taking into account limitations on dynamics of resources consumption during the scheduled period.

As a rule, each type of resource (e.g., human, material etc.) allocated for the project realization is distributed equally over the time. Provided that resource levels remain unchanged until the project is actually completed, we will schedule the project realization.

Let a dynamic model of a project development is described by a network diagram. The status of this system is determined by a schedule, or a set of arranged pairs $\left(T_{S}, t\right)$ on each activity of the project, where $T_{S}$ is the commencement of the activity, and $t$ is the duration of the activity.

\section{The problems' formulation}

Let $G=\{P, U\}$ be a network diagram described by the following pair of sets: $P$ is a set of events (nodes), $U$ is a set of activities $(\operatorname{arcs}) ; T_{p l}=\left\{T_{i j}, t_{i j},(i, j) \in U\right\}$ is a schedule of the project realization, where $T_{i j}$ is the scheduled commencement of the activity $(i, j), \mathrm{t}_{\mathrm{ij}}$ is the duration of the activity.

Let numerical function $C_{i j}=f_{i j}(t)$ correspond to each activity $(i, j) \in U$, where $t \in \tau_{i j}, \tau_{i j}$ being the range of determination for function. In addition $S_{d i r}$ and $C$ are given, where $S_{d i r}$ is the due date of the project completion, and $C$ is the budget of the project as a whole.

The problem is to determine schedule $T^{*}$ that minimizes the cost objective function

$$
C(T)=\sum_{(i, j) \in U} C_{i j}\left(t_{i j}\right),
$$


subject to

$T_{i j}+t_{i j} \leq S_{d i r}$ for all $(i, j) \in U, t_{i j} \in \tau_{i j}$

$\sum_{(i, j)=U} \frac{C_{i j}(t)}{t_{i j}} \cdot \delta_{i j}(t)-\frac{C}{S_{d i r}} \leq 0,0 \leq t \leq S_{d i r}$,

where

$$
\begin{aligned}
& \delta_{i j}(t)=1 \text { if } T_{i j} \leq t \leq T_{i j}+t_{i j}, \\
& \delta_{i j}(t)=0 \text { otherwise. } \\
& T_{i j} \geq t_{i}, i \in P,(i, j) \in U,
\end{aligned}
$$

where $t_{i}$ is the moment when the $i$-th event has occurred.

Problem (1-4) belongs to mathematical integer programming. Solving the problem is subject to one simplification. We take into account the large magnitude of the problem (the number of project activities can achieve several thousands). According to this assumption the solution of the initial problem is replaced by consecutive solutions of the following problems.

Problem I. It is required to determine a schedule of activities $\vec{t}^{*}=\left\{t_{i j}^{*},(i, j) \in U\right\}$, that minimizes the following objective function:

$$
C(\vec{t})=\sum_{(i, j) \in U} C_{i j}\left(t_{i j}\right)
$$

subject to

$$
T_{c r}\left(\vec{t}^{*}\right)-S_{d i r} \leq 0
$$

where $T_{c r}$ is the length of the critical path.

Problem II. Given a vector of durations $\vec{t}^{*}=\left\{t_{i j}^{*},(i, j) \in U\right\}$, it is required to determine vector $\vec{T}^{*}=\left\{T_{i j}^{*}, t_{i j}^{*},(i, j) \in U\right\}$ characterizing the commencement of each activity realization, which satisfies (1) and minimizes objective function:

$$
W(T)=\max _{t}\left[\sum_{(i, j) \in U} \frac{1}{t_{i j}} C_{i j}(t) \cdot \delta_{i j}(t)-\frac{C}{S_{d i r}}\right] .
$$

In other words, $W\left(T^{*}\right)=\min W(T)$.
The developed methods for solving Problems $I$ and II enable, in our view, to overcome difficulties associated with network large size. The solution is based on decomposition principle, i.e., decomposing a network project into network fragments, replacing them by a small number of aggregated activities, solving the problem for aggregated network project, and disaggregating the network diagram in order to schedule each project's activity. Earlier, the decomposition principle was widely used for calculation of time parameters of large size networks, e.g., in [1]. In this paper the same idea facilitates the solution of the problem by aggregating both time and cost parameters of a network.

\section{Example of fragmentary methods}

Sequence ("chain") of activities. This type of fragments has the following property: the beginning of realization for any (subsequent) activity (except for the first activity in a chain) is the end of realization of only one (previous) activity.

Designate all activities of the chain $\left(i, j_{1}\right),\left(j_{1}, j_{2}\right), \ldots,\left(j_{n}, j\right)$ and number them consecutively. Let $k=\overline{1, n}, i=j_{0}, j=j_{n+1}$, be given by the following numerical function:

$C_{k}=f_{k}(t)$, where $t \in \tau_{k}$.

Let $C_{i j}=f_{i j}(t)$ be alternative costs of realization of activity $(i, j)$, or the chain as a whole. This function has the following dependence on variants of realization for each activity of the chain:

$f_{i j}(t)=\min _{\sum t_{k} \leq t} \sum_{k=1}^{n} C_{k}\left(t_{k}\right), t \in[a, b]$, where $a=\sum_{k} \min \tau_{k}$,

$b=\sum_{k} \max \tau_{k}$.

Two methods can be applied to solve the problems. The first one is a graphical solution. The second one is a method of dynamic programming.

\subsection{Graphical solution}

In (7) minimum is taken on all sets $\left\{t_{1}, \ldots, t_{n}\right\}$, which in the sum do not surpass the given duration $t$ and $t_{k} \in \tau_{k}$. The function $f_{i j}(t)$ is monotonously nonincreasing and has the following technical and economic sense. The length of chain $t=\sum_{k=1}^{n} t_{k}$ and 
expenses $C=\sum_{k=1}^{n} C_{k}\left(t_{k}\right)$ unequivocally corresponds to each set $\left\{t_{1}, \ldots, t_{n}\right\}$. All sets $\left\{t_{1}, \ldots, t_{n}\right\}$ are represented by a set $M$ of points of a plane with axes of coordinates $C, t$. These sets can be numbered in a case of finite $\tau_{k}$.

Each point on $M$ represents a variant of realization for chain $(i, j)$. However, it is possible to remove from $M$ those variants with number $P$ (i.e., pairs $)\left(C_{P}, T_{P}\right)$ for each of which there exists a variant with number $q\left(C_{q}, T_{q}\right)$ (see Fig 1) if inequalities

$$
C_{q}<C_{P}, T_{q}<T_{P}
$$

are satisfied.

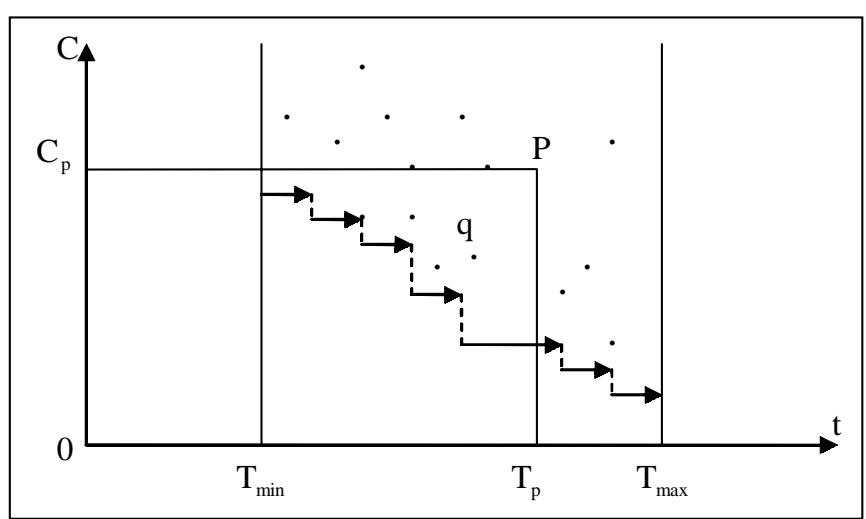

Fig 1. Variants of the project realization

After removal, the remaining points in coordinate plane $(C, t)$ allow us to construct function $f_{i j}(t)$. For this purpose we will renumber the points in ascending order of $t$ and draw a straight line from every point parallel to axis $t$ up to the coordinate $t$ of the subsequent point.

The suggested procedure of constructing function $f_{i j}(t)$ allows us only to bring it to light. However, the procedure is unpractical even for a small number of activities and variants. For example, for a chain with the number of activities equal to 10 and the number of variants equal to 3 it is required "to browse" about $3^{10}$ points for constructing function $f_{i j}(t)$.

\subsection{Method of dynamic programming}

For constructing function $f_{i j}(t)$ we will use a method of dynamic programming [2]. Herein the calculation of $f_{i j}(t)$ for any $\mathrm{t}$ is carried out by consecutive calculation of value $t_{k} \in \tau_{k}$ on the ${ }_{n}{ }^{k}$-th step $(k=\overline{1, n})$ to obtain the minimal sum value $\sum_{k=1}^{n} C_{k}\left(t_{k}\right)$ subject to $\sum_{k=1}^{n} t_{k} \leq t$
Let $C^{*}\left(x_{N-1}\right)=\min _{\sum_{k=1}^{N-1} t_{k} \leq x_{N-1}} \sum_{k=1}^{N-1} C_{k}\left(t_{k}\right)$

be expenses corresponding to the optimal allocation of $x_{N-1}$ on the $(N-1)$-th step, $\left(x_{1}^{*}, \ldots, x_{N-1}^{*}\right)$ are variants of allocation of the accumulated sum $\sum_{k=1}^{N-1} t_{k}$ up to the $(N-1)$ th step. Thus, the recursive equation, which is satisfied by optimal allocation after $N$ steps, is as follows:

$$
C^{*}\left(x_{N}\right)=\min _{x_{N-1}+t_{N} \leq x_{N}}\left[C^{*}\left(x_{N-1}\right)+C_{N}\left(t_{N}\right)\right],
$$

$N=2, \ldots, n$.

\section{Models for budget allocation for PERT-COST type projects}

The problem of optimal control for a PERT-COST network project is solved. At the upper level - the company level - the problem focuses on minimizing the total budget assigned to the project in order to meet the pregiven project's due date under pregiven probability constraint. At the second level - the project level - the problem's solution boils down to an optimal budget reassignment among the project's activities. At the lower level - activity level - thee planned starting moments for each activity entering the project, have to be determined. Thus, the lower hierarchical level solves scheduling problems.

The project under consideration is a PERT-COST type network project $G(N, A)$ with a deterministic structure and activities of random duration. For each activity $(i, j) \subset G(N, A)$ the budget $c(i, j)$ assigned to that activity enters parametrically in the corresponding probability density function (p.d.f.).

The suggested method of the problem's solution is as follows. A subsidiary problem $[3,1]$ to maximize the system's reliability to accomplish the project at the pregiven due date $D_{G}$ for a pre-given budget amount $C_{G}$ assigned to the project, has to be solved. Here the optimized variables are values $c(i, j)$ assigned to each activity, subject to

$$
\begin{aligned}
& c_{\text {min }}(i, j) \leq c(i, j) \leq c_{\text {max }}(i, j), \\
& \sum_{\{i, j\}} c(i, j)=C_{G} .
\end{aligned}
$$

We suggest to solve the subsidiary problem by means 
of numerous monotonously increasing values $C_{G}$ by a pregiven search step $C_{G}+\delta C \Rightarrow C_{G}$, until the project's reliability $\operatorname{Pr}\left\{T_{G} \leq D_{G}\right\}$ starts to satisfy

$$
\underset{C_{G},\{c(i, j)\}}{\operatorname{Max}} \operatorname{Pr}\left\{T_{G} \leq D_{G}\right\} \geq p^{*} .
$$

The first value $C_{G}$ which satisfies the last inequality for pregiven $D_{G}$ and $p^{*}$, is taken as the quasi-optimal solution of the problem under consideration. Note that $T_{G}$ is a random time duration to accomplish the project with optimal value $C_{G}$.

The general idea of solving the subsidiary problem is based on implementing the global search method by means of simulating the initial search point using the Monte-Carlo method (see, e.g. [3]). A local random search [3] is carried out from each initial point in order to obtain the local optimal value. The combination: simulating the initial point $\Rightarrow$ optimizing the objective by means of a local random search method, is carried out for numerous initial points in order to choose the optimal value from the representative statistics. Thus, the outlined above subsidiary problem is dealt with by means of the Monte-Carlo method. However, simplified heuristic algorithms for solving that problem are available as well $[1,3,4]$.

\section{Conclusions}

1. The problem of the optimal scheduling of the project activities is considered and solved. The solution is based on decomposition principle, i.e., decomposing a network project into network fragments, replacing them by a small number of aggregated activities, solving the problem for aggregated network project, and disaggregating the network diagram in order to schedule each project's activity.

2. Multiple usage of the decomposition principle enables us to improve consecutively the schedule for an initial network diagram up to the moment of reaching a local extremum.

3. A budget reallocation procedure among PERTCOST activities is suggested. The procedure is based on the combination of a heuristic method and a simulation model.

\section{Acknowledgement}

This research has been partially supported by the Paul Ivanier Center on Robotics and Production Management, Ben-Gurion University of the Negev.

\section{References}

[1] Gonik, A. Planning and Controlling Multilevel Stochastic Projects, Ph.D. Thesis, Ben-Gurion University of the Negev, Beer-Sheva, 1995.

[2] Taha, H. A. Operations Research: An Introduction, Collier Macmillan, New York, 1997.

[3] Golenko-Ginzburg, D. A two-level decision-making model for controlling stochastic projects. International Journal of Production Economics, 32, 1993, p. 117-127.

[4] Rutkauskas, A. V.; Stankevičienè, J. Formation of an investment portfolio adequate for stochasticity of profit possibilities. Journal of Business Economics and Management, Vol IV, No 1, 2003, p. 3-12. 